\title{
Daily rhythm of aggregation in the haematophagous bug Triatoma infestans (Heteroptera: Reduviidae)
}

\author{
SA Minoli/*/+, S Baraballe*, AN Lorenzo Figueiras*
}

Institut de Recherche sur la Biologie de l'Insecte, Université François Rabelais, Tours, France *Departamento de Biodiversidad y Biología Experimental, Facultad de Ciencias Exactas y Naturales, Universidad de Buenos Aires, Pabellón II, $4^{\circ}$ Piso, C1428EHA

Buenos Aires, Argentina

Triatomine bugs show a temporal modulation of many activities. Here, we analyse the daily modulation of the aggregation behaviour of Triatoma infestans larvae and its chronobiological basis. In the laboratory, groups of six bugs were released over an experimental arena during six consecutive days, where their aggregation behaviour was quantified every hour. When submitted to a 12/12 h photoperiod (L/D), the larvae of T. infestans exhibited a cyclic pattern of aggregation with a $24 \mathrm{~h}$ period, evincing the existence of a daily rhythm of aggregation in this species. Bugs exhibited the maximum aggregation tendency at the end of the scotophase (7:00 h), moment in which they naturally search for refuges. The minimum aggregation (i.e. maximal dispersion) was observed during the last part of the photophase and beginning of the scotophase (15:00 to 1:00 h). This cyclic pattern disappeared when constant conditions of illumination $(L / L)$ or darkness $(D / D)$ were imposed to the bugs, suggesting the absence of an endogenous circadian control of this behaviour. Insects submitted to $L / L$ and $D / D$ photoperiods presented lower global levels of aggregation than those submitted to L/D conditions. The lack of an endogenous control and the relevance of light cycles as a synchronization signal are discussed as the temporal modulation of this behaviour might play an important role in the nocturnal habits of this species.

Key words: triatomines - daily rhythm - aggregation behaviour

Daily rhythms have been observed in a wide variety of behavioural, physiological, and metabolic processes in many insects (Saunders 1982). Individuals maintained under natural or artificial cycles of light and/or temperature express daily spontaneous activity patterns, primarily restricted to a particular period during the day.

Triatomine bugs, vectors of the Chagas disease, are usually referred as having typically nocturnal habits. Remarkably, their life exhibits a high degree of temporal organization and different processes have been described to be temporally modulated. Most species of this group of blood-sucking insects leave their refuges at the beginning of the night and display the major part of their activities (e.g. host search, feeding, dispersion, etc.) in discrete temporal windows during this dark period (Ampleford \& Davey 1989, Lazzari 1991, 1992, McEwen \& Lehane 1993; Lorenzo Figueiras et al. 1994, Roca \& Lazzari 1994, Lorenzo \& Lazzari 1998, Guarneri et al. 2002, Minoli \& Lazzari 2003, Barrozo et al. 2004a). Before sunrise, usually after a blood meal has been obtained, they return to their refuges aimed by chemical cues released by themselves and conspecifics (Lorenzo \& Lazzari 1996), and possibly guided by their

\footnotetext{
+Corresponding author: minoli@bg.fcen.uba.ar Received 3 October 2006 Accepted 12 March 2007
}

thermal and hygric preferences (Minoli \& Lazzari 2003). During the daylight hours they remain aggregated inside their shelters, usually in akinetic state (Lorenzo Figueiras et al. 1994). This temporal dynamics of occupation of shelters involves a high degree of association between individuals and with their refuges, as bugs spend a great part of their lives inside one of them.

Triatoma infestans is the main vector of the Chagas disease in southern South America, mainly due to its domestic habits. This species is often found inside human dwellings, mostly in the sleeping rooms, where they occupy different specific shelters (e.g. wall crevices, straw roofs, under mattresses, etc.). Normally, infested houses in endemic areas exhibit high occupancy levels of these bugs, for what the availability of refuges could often be limited. Shelters are thus usually occupied by more than one individual of the same species. As a consequence of the temporal exploitation and of the potentially limited availability of suitable shelters, $T$. infestans spends plenty of time sharing shelters with other individuals, in close contact with both, conspecifics and the substrate. However, this co-occupancy of shelters could be just a consequence of a preference for similar shelters or, on the other hand, it could be mediated by the presence of cues released by other individuals, process that could be regarded as a true aggregation. In this respect, many reports show the aggregation behaviour of triatomine bugs mediated by assembling pheromones present in the faeces, both in an intra and interspecific manner (Lorenzo Figueiras \& Lazzari 1998, Pires et al. 2002). Moreover, the temporal change of the aggregation behaviour of $T$. infestans along days has already been analyzed (Lorenzo Figueiras \& Lazzari 2000). These authors showed that the mean aggregation levels of these 
bugs augmented as the post-feeding time does, but they did not analyse how aggregation might change during day. Remaining inside shelters during the daylight hours could become essential for triatomines survival, as normally their hosts can play the role of predators when they are awake. However, although changes in the aggregation levels of triatomines during day might help in the reinforcement of their nocturnal habits, no attempts to analyse their daily aggregation dynamics were made so far.

Here, we analyze the daily aggregation dynamics of T. infestans adding no other external cues than the photoperiod. The objective of this work is to describe the daily variation of aggregation in these haematophagous bugs and to uncover possible endogenous rhythmic components of this behaviour.

\section{MATERIALS AND METHODS}

Insects - Larvae of the 5th instar of T. infestans, reared in the laboratory at a temperature of $27 \pm 2^{\circ} \mathrm{C}$ and $60 \pm 20 \%$ relative humidity, under a $12 \mathrm{~h}$ light (08:00 to 20:00 h) - $12 \mathrm{~h}$ darkness (20:00 to 08:00 h) photoperiod, were used throughout the experiments. All previous instars were fed once a week on live hens, and once they become 5th instar larvae were maintained unfed for 10-20 days before being used in the experiments. This starvation level was selected as Lorenzo Figueiras and Lazzari (2000) showed that only after eight days after feeding bugs began to aggregate around dry faeces.

Experimental device - An experimental glass arena (length: $39 \mathrm{~cm}$, width: $9.25 \mathrm{~cm}$, height: $3 \mathrm{~cm}$ ) with a cover lid was placed in a thermostatized room $\left(25^{\circ} \mathrm{C}\right)$. The floor of the arena was covered with filter paper, which was replaced between replicates to eliminate putative cues deposited by the animals. Each time, six bugs were released simultaneously in the arena and their positions were individually recorded as vectors with origin at a reference point placed arbitrarily at one end of the arena. The position of each insect was so registered every hour during six consecutive days with the aid of an infraredsensitive video camera, connected to a video recorder controlled by a PC. Before the beginning of the recording, the insects remained in the arena for $4 \mathrm{~h}$ to familiarize to the experimental situation. Insects' positions were then digitalized hourly from the video recordings by means of a software developed ad hoc in our laboratory. The distances between the six bugs of each group in the arena (Fig. 1, 15 values each hour) were calculated (pixel units) every hour and combined to calculate an aggregation index as:

$$
A I_{(t)}=\frac{1}{\sum_{i=1}^{15} m_{i}} \cdot 10000
$$

where $A I_{(t)}$ is the aggregation index at time $t$, and $m_{i}$ is the $i$ distance between two bugs. The parameter $i$ attains entire values between 1 and 15. An additional factor of 10000 was multiplied to avoid small numbers in the graphs. The higher the AI becomes, the lower the distance between bugs is. The distance $m_{i}$ was calculated as:

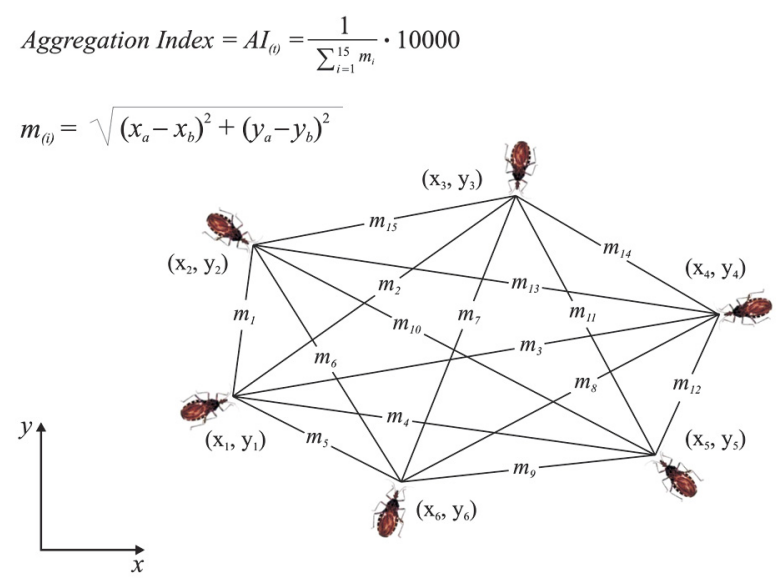

Fig. 1: diagram showing how the the AI estimates the aggregation levels of the bugs at each moment using the 15 linear distances between them. $A I_{(t)}$ is the AI time $t ; m_{i}$ is the $i$ distance between two bugs; $i$ includes entire values between 1 and 15 ; a factor of 10000 was added to the formula to avoid small numbers in the graphs; $x_{a}$ and $x_{b}$ are the abscissas and $\mathrm{y}_{a}$ and $\mathrm{y}_{b}$ the ordinates of the position of the two insects over the arena.

$$
m_{(i)}=\sqrt{\left(x_{a}-x_{b}\right)^{2}+\left(y_{a}-y_{b}\right)^{2}}
$$

where $x_{a}$ and $x_{b}$ are the abscissas and $\mathrm{y}_{a}$ and $\mathrm{y}_{b}$ the ordinates of the position of the two considered insects, respectively.

The AI was calculated each hour, during six consecutive days, for three experimental series with different illumination regimes: (1) L/D or 12/12 h: insects received $12 \mathrm{~h}$ of light (60 lux) and $12 \mathrm{~h}$ of darkness ( 0 lux) each day; (2) L/L or $24 / 0 \mathrm{~h}$ : the light (60 lux) was continuously turned on; and (3) D/D or 0/24 h: the behaviour of the bugs was analyzed in constant darkness (0 lux). Eight replicates of each experimental series were carried out, comprising a total of 144 insects.

Statistical analysis - We checked the occurrence of periodical components in the time series of the AI of bugs tested under $\mathrm{L} / \mathrm{D}, \mathrm{L} / \mathrm{L}$, and $\mathrm{D} / \mathrm{D}$ conditions by the LombScargle periodogram procedure (Lomb 1976, Scargle 1982) using the software LSP 2.2 (created by R Refinetti, freely available in the internet). The mean aggregation levels of bugs submitted to the different photoperiods were compared by means of a one-way Anova $(\alpha=0.05)$, after verifying the assumptions of normality and homogeneity of variances of the data. Tukey HSD post-hoc comparisons were made when necessary (Zar 1984).

\section{RESULTS}

Daily rhythm of aggregation - In Figs 2A-F we depict the mean aggregation behaviour $(n=8)$ of bugs submitted to the three different illumination regimes along the six days, and their statistical analyses. In Fig. 3 we show the daily dynamics of aggregation of the same groups of insects, by averaging the AIs of a particular time of the day of the six different days $(n=6)$. Insects 


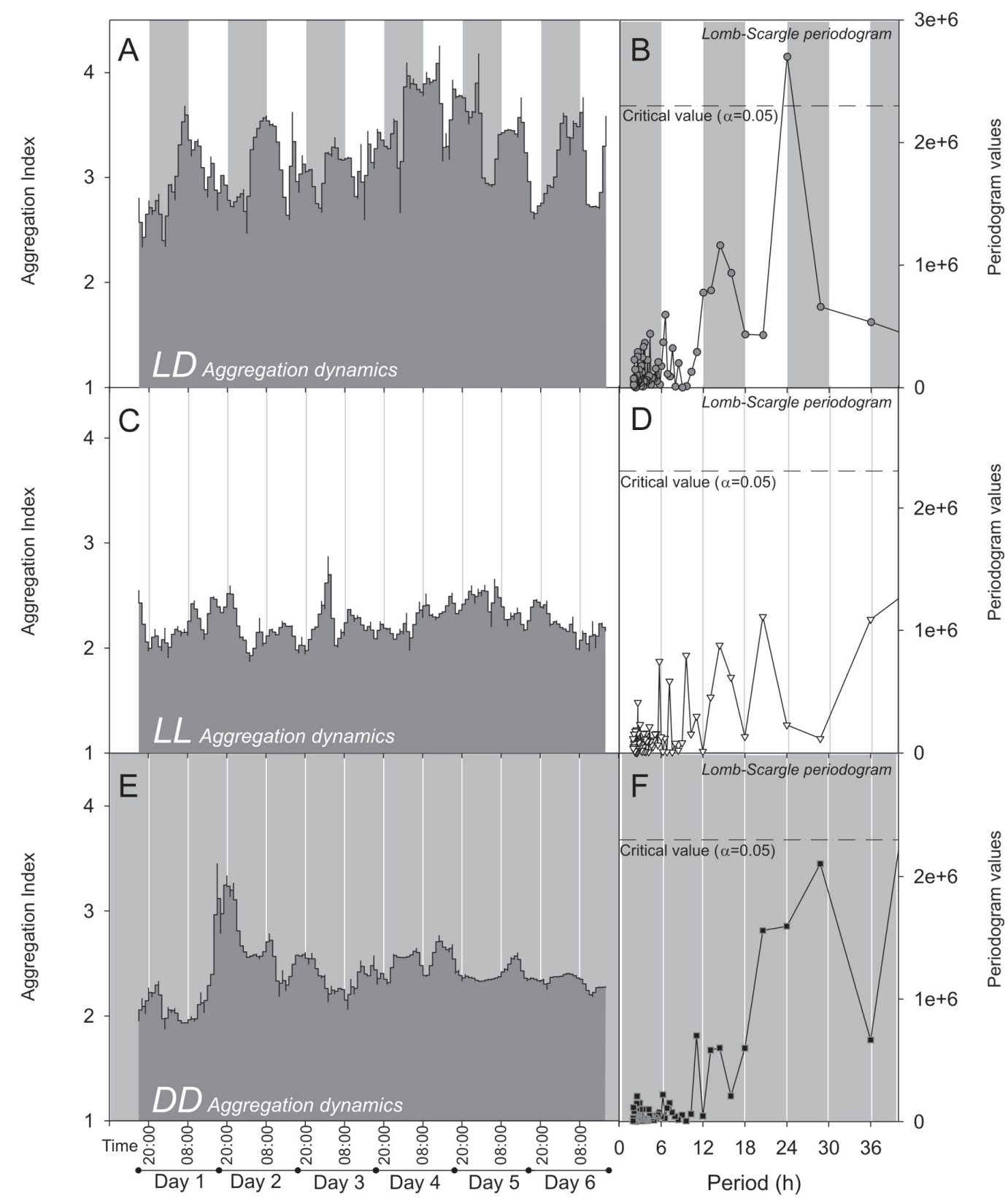

Fig. 2: temporal dynamics of aggregation (AI) of triatomines. The pairs of subfigures A-B, C-D and E-F show the temporal dynamics of aggregation of bugs and their temporal statistical analyses (Lomb-Scargle Periodograms) when the three different photoperiods where imposed, respectively (i.e. $\mathrm{L} / \mathrm{D}, \mathrm{L} / \mathrm{L}$ and $\mathrm{D} / \mathrm{D})$. A daily, rhythm of aggregation was corroborated for bugs tested under $\mathrm{L} / \mathrm{D}$ conditions. No cycle was observed in bugs submitted to $\mathrm{L} / \mathrm{L}$ and $\mathrm{D} / \mathrm{D}$. The figures represent the average $\mathrm{AI}$ of eight replicates for each photoperiod $(\mathrm{n}=8)$ during the six days. The periodograms exhibit the most repeated period of each temporal series and its statistical analysis (dashed lines indicate the critical level at $\alpha=0.05$ ).

submitted to L/D (i.e. 12/12 h light/darkness photoperiod) showed that the aggregation process in triatomines is not temporally static, but instead, it is a dynamic process that changes with the time of the day (Fig. 2A, B). We found that during the scotophase, triatomines exhibited a slow but continuous tendency to increase their aggregation levels (Fig. 3A), reaching a maximum just before the beginning of the photophase, i.e. at 7:00 h. Subsequently, after lights were turned on, bugs gradually began to disperse, reaching the minimum aggregation at 15:00 h and keeping it until midnight. The daily rhythm of aggregation was demonstrated throughout the six experimental days of the L/D series, obtaining a statistically significant period of $23.99 \mathrm{~h}$ (Lomb-Scargle Periodogram, $p<0.05$; Fig. 2B). Contrarily, when constant conditions of illumination (L/L) or darkness (D/ D) were imposed to bugs (Figs 2C, E, 3A), no rhythmical behaviour was evinced. The Lomb-Scargle Periodograms did not reveal significant periodical elements in these series ( $p>0.05$ in both cases; Fig. 2D, F). The non-persistence of the daily rhythm of aggregation in the absence of external cues suggests that there would be no endogenous clocks controlling the aggregation behaviour in these insects. 


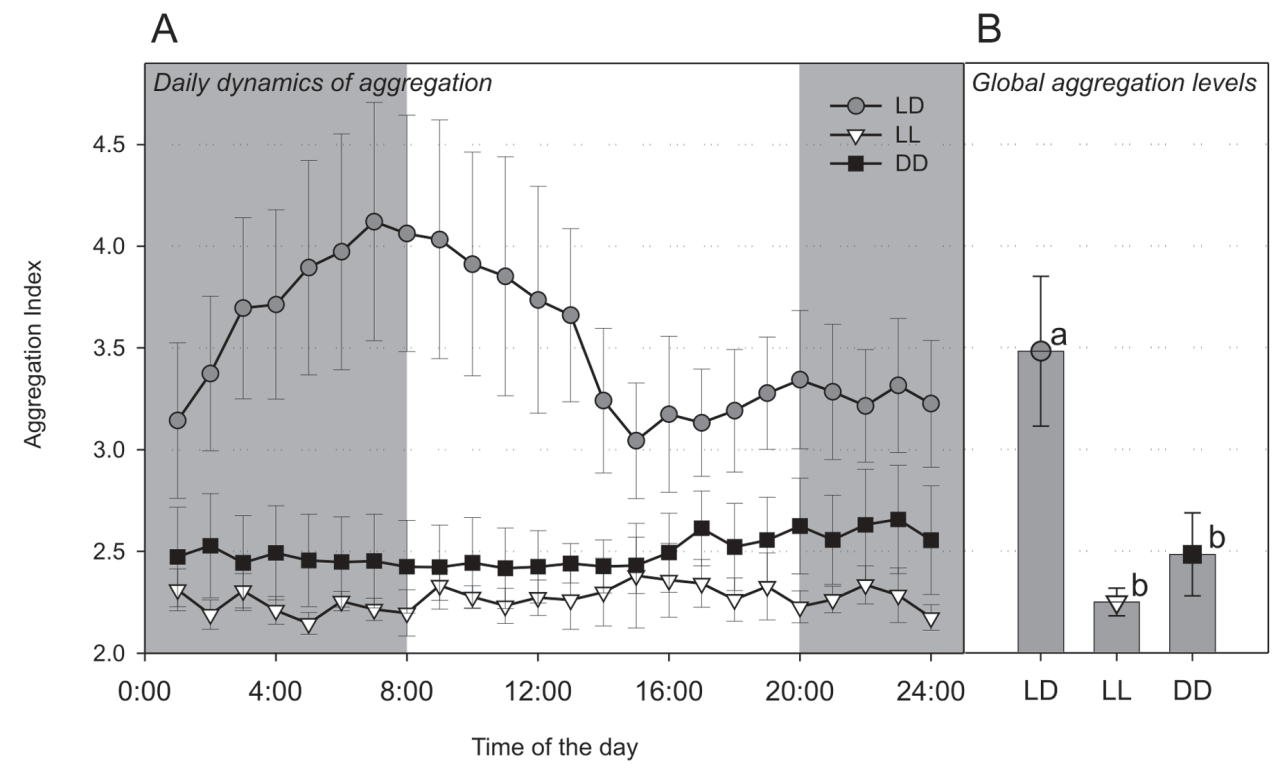

Fig. 3A: daily dynamics of aggregation (AI) of triatomines. Bugs submitted to L/D expressed a daily cycle of aggregation with a slow but continuous tendency to disperse during the day and to re-aggregate during the night. As a consequence, the maximum aggregation tendency is evinced at the end of the night $(7: 00 \mathrm{~h})$, moment in which insects search for refuges. Each point represents the mean AI of the six experimental days $(\mathrm{n}=6$, SE in bars) at a particular time of the day; B: global aggregation levels (AI) of triatomines. Bugs submitted to L/D presented a continuously higher tendency to aggregate than the other two groups, evinced in a higher global aggregation level.

Aggregation levels - We compared the global aggregation tendency of bugs submitted to the three different illumination regimes. We found that the photoperiods assayed affected the aggregation behaviour of this species in different ways (Fig. 3B; One-way-Anova, $\left.\mathrm{F}_{(2,21)}=7.09, \mathrm{p}<0.005\right)$. Post-hoc comparisons revealed that when light/darkness cycles were imposed to the insects (i.e. L/D), their global aggregation level was higher than when they were exposed to constant illumination (L/L; Tukey HSD Test, $\mathrm{p}<0.01$ ) or constant darkness (D/D; Tukey HSD Test, $\mathrm{p}<0.03$ ). No differences were found between the aggregation of bugs submitted to $\mathrm{L} / \mathrm{L}$ and D/D (Tukey HSD Test, $p$ > 0.78).

\section{DISCUSSION}

Triatomine bugs remain an important part of their lives aggregated inside their refuges (e.g. crevices, hollows, cracks or fissures). Two main components of the aggregation behaviour might be acting together sustaining this behaviour: the tendency of bugs to remain in close contact with the substrate (thigmotaxis) and the trend to remain in close proximity to other individuals (conspecific aggregation). Many advantages as a result of the aggregation behaviour of individuals have been classical proposed within the animal kingdom (e.g. defence against predators, decrease in water or heat loss, increase in mating probability, etc). Moreover, as coprophagy, cannibalism, and hemolinphagy are alternative ways for triatomine nymphs to obtain blood meals (Schaub et al. 1989), these processes might increase the aggregation tendency of these bugs. In this work we analyzed the aggregation behaviour of $T$. infestans when no other cues than those offered by their congeners' body were present in the experimental situation, i.e. no refuges or odours added. Fifth instars larvae of this species were used throughout experiments as they are easily localized in the experimental device (smaller larvae are not detected with the camera during scotophases) and do not release sexual pheromones (as adults do) that stick to the floor and walls of the arena might modify the aggregation behaviour as measured in our experiments. However, although not studied in this work, the presence of odours released by adults via their Brindley's and/or metathoraxic glands might increase even more the tendency of these insects to aggregate.

As expected for the marked temporal organization of activities these insects present, we found that the aggregation behaviour of this species varies along day (Figs 2A, B, 3A). However, no endogenous periodical components were found to govern this behaviour, but instead an external cue such as the photoperiod showed to be a relevant factor in its modulation (Figs. 2C, D, E, F, 3A). This result is not less than surprising, as these bugs have proven to have one or more biological clocks controlling many of their activities (Ampleford \& Steel 1982, Constantinou 1984, Ampleford and Davey 1989, Lazzari 1991, 1992, Lorenzo \& Lazzari 1998, Reisenman et al. 1998, 2002, Lorenzo Figueiras \& Lazzari 2000, Minoli \& Lazzari 2003, Barrozo et al. 2004a). Circadian control of behaviour provides animals a temporal orientation independently from environmental cues for the occupation of temporal niches or in the co-ordination of activities between individuals and species (Aschoff 1989). However, the results here presented seem to highlight the relevance of the photoperiod as a natural clock.

The daily dynamics of aggregation behaviour exhibited by $T$. infestans presented two opposite tendencies that seem to be associated directly to the presence of light (Fig. 3A): insects presented an increasing tendency 
to disperse along the photophase and to aggregate during the scotophase. In this way, bugs would attain their maximum dispersion tendency just before the sunset, moment in which they spontaneously become more active (Lazzari 1992, Lorenzo Figueiras et al. 1994, Lorenzo \& Lazzari 1998), and began the food-search behaviour among other activities. Contrarily, just before sunrise, the bugs would present a marked predisposition to re-aggregate, helping in the refuge-search behaviour normally expressed at this moment. In this sense, we propose that the daily aggregation behaviour evinced by $T$. infestans may reinforce the nocturnal habits of this species.

Although the AI as it is calculated in this work is somehow related with the locomotion activity of bugs, these two processes are quite independent from each other. Intuitively we are likely to think that a decrease in locomotion activity will unambiguously lead to an increase in aggregation and vice-versa. However, this assertion is incorrect, as the locomotion activity alone is not enough for explaining temporal changes in AI evinced by bugs. In this way, a low activity level might result in both, a high AI if bugs are immobile and near each other, or a low AI if they are immobile but far away from each other. The same reasoning may be applied for a high activity level, as bugs might move collectively along the arena (high AI) or independently (low AI). Inversely, a particular AI informs us unequivocally about the proximity between bugs independently of their activity level. A high AI evinces unambiguously that bugs are near each other, no matter if they are still or moving around the arena. Moreover, the daily rhythm of aggregation evinced in this work does not match with the bimodal daily rhythm of activity described by Lazzari (1992) for this species. If the activity levels of bugs were to be governing the changes in AI, we would expect a correlation between the two variables. As this is not the case, we can be sure that what we are analysing in this work is effectively the aggregation dynamics of these bugs, and not their activity pattern.

The global levels of aggregation during the overall experimental time varied with the photoperiods (Fig. 3B). Surprisingly, the maximal levels of AI were verified when the L/D photoperiod was imposed and not when constant darkness was presented, as it would be expected if the slow but continuous tendency of bugs to aggregate during night and disperse during day observed in the previous section (Fig. 3A) was still to occur. None of the two assumptions were corroborated. We could speculate that changes in the light intensity are indispensable for aggregation to occur. However, no direct evidences are available to support this assertion, for what it remains uncertain.

This work contributes with others that intend to highlight the importance of the time of day in which the experiments are carried out. As it is pointed by Barrozo et al. (2004b) and as it is shown in this report, static measures of animal behaviour may misestimate the real values if time is not taken into account. Thus, the interpretation of experimental studies can be seriously compromised unless they are repeated at different moments of the circadian cycle to determine if the responses vary along the day.

\section{REFERENCES}

Ampleford EJ, Davey, KG 1989. Egg laying in the insect Rhodnius prolixus is timed in a circadian fashion. Insect Physiol 35: 183-187.

Ampleford EJ, Steel C 1982. Circadian control of ecdysis in Rhodnius prolixus (Hemiptera). J Comp Physiol 147: 281-286.

Aschoff J 1989. Temporal orientation: circadian clocks in animals and humans. Animal Behavior 37: 881-896.

Barrozo RB, Minoli SA, Lazzari CR 2004a. Circadian rhythm of behavioural responsiveness to carbon dioxide in the bloodsucking bug Triatoma infestans (Heteroptera: Reduviidae). J Insect Physiol 50: 249-254.

Barrozo RB, Schilman PE, Minoli SA, Lazzari CR 2004b. Daily rhythms in disease-vector insects. Biol Rhythms Res 35: 79-92.

Constantinou C 1984. Circadian rhythm of oviposition in the blood sucking bugs, Triatoma phyllosoma, T. infestans and Panstrongylus megistus (Hemiptera: Reduviidae). J Interdisc Cycle Res 15: 203-212.

Guarneri AA, Lazzari CR, Diotaiuti L, Lorenzo MG 2002. The effect of relative humidity on the behaviour and development of Triatoma brasiliensis (Hemiptera, Reduviidae). Physiol Entomol 27: 142-147.

Lazzari CR 1991. Circadian rhythm of egg hatching in Triatoma infestans (Hemiptera: Reduviidae). J Med Entomol 28: 740-741.

Lazzari CR 1992. Circadian organization of locomotion activity in the haematophagous bug Triatoma infestans. J Insect Physiol 38: 895-903.

Lomb NR 1976. Least-squares frequency analysis of unequally spaced data. Astrophys Space Sci 39: 447-462.

Lorenzo Figueiras AN, Lazzari CR 1998. Aggregation behaviour and interspecific responses in three species of Triatominae. Mem Inst Oswaldo Cruz 93: 133-137.

Lorenzo Figueiras AN, Lazzari CR 2000. Temporal change of the aggregation response in Triatoma infestans. Mem Inst Oswaldo Cruz 95: 889-892.

Lorenzo Figueiras AN, Kenigsten A, Lazzari CR 1994. Aggregation in the haematophagous bug Triatoma infestans: chemical signals and temporal pattern. J Insect Physiol 40: 311-316.

Lorenzo MG, Lazzari CR 1996. The spatial pattern of defecation in Triatoma infestans and the role of faeces as a chemical mark of the refuge. J Insect Physiol 42: 903-907.

Lorenzo MG, Lazzari CR 1998. Activity pattern in relation to refuge exploitation and feeding in Triatoma infestans (Hemiptera: Reduviidae). Acta Trop 70: 163-170.

McEwen PK, Lehane MJ 1993. Factors influencing flight initiation in the triatomine bug Triatoma infestans (Hemiptera: Reduviidae). Insect Sci Applications 14: 461-464.

Minoli SA, Lazzari CR 2003. Chronobiological basis of thermopreference in the haematophagous bug Triatoma infestans. J Insect Physiol 49: 927-932.

Pires HH, Lorenzo MG, Diotaiuti L, Lazzari CR, Lorenzo Figueiras AN 2002. Aggregation behaviour in Panstrongylus megistus and Triatoma infestans: inter and intraspecific responses. Acta Tropi 81: 47-52. 
Reisenman CE, Insausti TC, Lazzari CR 2002. Light-induced and circadian changes in the compound eye of the haematophagous bug Triatoma infestans (Hemiptera: Reduviidae). J Exp Biol 25: 201-210.

Reisenman CE, Lazzari CR, Giurfa M 1998, Circadian control of photonegative sensitivity in the haematophagous bug Triatoma infestans. J Comp Physiol A 183: 533-541.

Roca MJ, Lazzari CR 1994. Effects of relative humidity on the haematophagous bug Triatoma infestans: hygropreference and eclosion success. J Insect Physiol 40: 901-907.
Saunders DS 1982. Insect Clocks, 2nd ed., Pergamon Press, Oxford.

Scargle JD 1982. Studies in astronomical time series analysis. II - Statistical aspects of spectral analysis of unevenly spaced data. Astrophysical J 302: 757-763.

Schaub GA, Böker CA, Jensen C, Reduth D 1989. Cannibalism and coprophagy are modes of transmission of Blastocrithidia triatomae (Trypanosomatidae) between triatomines. J Protozool 36: 171-175.

Zar JH 1984. Biostatistical Analysis, Prentice-Hall, New Jersey. 